\title{
Quality of life under the COVID-19 quarantine
}

\author{
Lara N. Ferreira ${ }^{1,2,3}$ [D $\cdot$ Luís N. Pereira ${ }^{1,2,3}$ [D $\cdot$ Maria da Fé Brás ${ }^{1,4} \cdot$ Kateryna llchuk $^{5}$
}

Accepted: 24 November 2020 / Published online: 2 January 2021

(c) Springer Nature Switzerland AG 2021

\begin{abstract}
Background COVID-19 has spread rapidly throughout the world, causing thousands of illnesses and deaths. To fight this pandemic, almost all governments and health authorities have focused on prevention. In March or April, most countries' officials imposed home quarantine and lockdown measures nationwide.

Purpose This study sought to assess health-related quality of life (HRQoL) and anxiety levels among people in Portugal under mandatory home quarantine due to the COVID-19 pandemic. The results were compared to the general Portuguese population's HRQoL before the COVID-19 outbreak. This research also aimed to understand the factors that can influence the respondents' HRQoL.

Methods A sample of Portugal's population quarantined at home $(n=904)$ filled in an online survey comprising the Generalized Anxiety Disorder 7-item and the EQ-5D-5L and other questions about sociodemographic characteristics, feelings, duties and activities during the quarantine. The sample was weighted to mirror the general population's gender, age and education. Descriptive analyses and correlation coefficients were used to evaluation the respondents' anxiety and HRQoL. Generalised linear models were estimated to identify determinants of HRQoL during the COVID-19 quarantine.

Results The results show that individuals quarantined at home reported higher anxiety and lower HRQoL levels and that people with more anxiety tended to have a lower HRQoL. Females and elderly individuals experienced the highest levels of anxiety and poorest HRQoL. In addition, HRQoL during the quarantine can be explained by various occupational and attitudinal variables, as well as sociodemographic variables.

Conclusion Individuals' mental health should be taken into consideration during pandemics or other emergency situations. Anxiety and other factors can decrease people's HRQoL, in conjunction with the pandemic's social and economic consequences.
\end{abstract}

Keywords Anxiety $\cdot$ COVID-19 $\cdot$ EQ-5D-5L $\cdot$ GAD-7 $\cdot$ Pandemic $\cdot$ Quality of life

\section{Introduction}

COVID-19 has had a great impact on many levels. This disease is caused by a new strain of coronavirus called severe acute respiratory syndrome coronavirus 2 that spread

Lara N. Ferreira

lnferrei@ualg.pt

1 Universidade do Algarve - ESGHT, Faro, Portugal

2 Centre for Health Studies and Research of the University of Coimbra (CEISUC), Coimbra, Portugal

3 Research Centre for Tourism, Sustainability and Well-Being (CinTurs), Faro, Portugal

4 Centre for Tourism Research, Development and Innovation (CiTUR), Faro, Portugal

5 International Centre on Aging (CENIE), Salamanca, Spain rapidly throughout China, sparking concerns about a global pandemic. By the end of May 2020, it had spread outside China, crossing international borders all over the world, with $5,459,528$ confirmed cases and 345,994 deaths-a mortality rate of about 6.3\% [1]. In Europe, as of 26 May, 1,843,581 cases had been reported, and the numbers continued to grow. As of that date, Europe had 168,308 confirmed infectionrelated deaths, corresponding to a mortality rate of $9.1 \%$, according to data published by the European Centre for Disease Prevention and Control [1]. Portugal had 31,007 reported cases and a lower fatality rate (i.e. $4.3 \%$ ).

With the pandemic evolving even more rapidly in the United States (US), Brazil, and India, concerns arose that these numbers would get even worse. This infectious disease is particularly lethal among the elderly, with even higher fatality rates among this age group (i.e. $16.7 \%$ in Portugal as 
of 26 May). Because Europe has a high proportion of older people, most European governments have taken actions to protect individuals more than 70 years old.

This emerging infectious disease pandemic has significant implications for clinical and public healthcare, as well as having a major impact on economic trends in almost all sectors. The measures imposed in almost all countries to deal with the pandemic are sending the entire world spiralling into an extensive crisis and thus into a deep global recession. The International Monetary Fund's [2] recently published estimates indicate that each month that non-essential services are closed will translate into a 3\% drop in the relevant countries' annual gross domestic product.

This COVID-19 pandemic has placed unprecedented pressure on societies and healthcare systems worldwide. The situation requires internationally unified, cogent and collective initiatives that mitigate its escalating effects [3]. As no vaccine or effective treatment is yet available for this disease, almost all governments and health authorities have focused on prevention. Public health officials' primary preventive advice has concentrated on hand hygiene and social distancing [3].

Each day, more countries are closing their borders, repatriating their citizens, discouraging tourism and banning mass gatherings [3]. Air travel has been largely suspended not only between continents but also within continents and inside countries as shown, for example, by the decrease in air travel within Europe and inside various European countries since mid-March 2020. Despite these efforts and worldwide precautionary measures, the number of cases and deaths continues to increase around the globe.

Many governments have imposed measures of social isolation that, in many countries, have rapidly evolved into home isolation and quarantine, with serious consequences for entire populations and economies. By 3 April, approximately 4 billion people worldwide-roughly half of all humanity - were engaging in home isolation and quarantine.

As Portugal has a high proportion of elderly individuals [4] and its National Health Service has been in crisis for years, the Portuguese government decided to implement rapid measures to prevent the National Health Service's collapse and a high mortality rate. On 16 March, all schools were closed, following the closure of all universities 1 week previously. On 18 March, the government declared a state of emergency, putting into place various restrictive measures that included mandatory confinement of patients with COVID-19 and a special duty of protection of at-risk groups (i.e. those over 70 years of age and with chronic diseases).

Other restrictions were a general duty of home confinement (i.e. home quarantine), mandatory telework whenever possible, provision of public services primarily through digital means and restrictions on road traffic and other modes of transport within the country. In addition, the government banned religious celebrations, closed specific types of establishments and reduced the activity of others (e.g. restaurants, bars and shops) and prohibited collective physical exercise [5]. Further measures were adopted, such as free public transport to avoid exchanges of money, restricted access to beaches and parks and outdoor exercise only alone or with someone from the same household. Most borders were closed (i.e. only a few land border points between Portugal and Spain were open with restricted access), and flights were suspended, among other restrictions [5, 6].

The state of emergency lasted until 2 May. During this period, even more extremely restrictive measures prohibited the circulation of people between cities without strong justification during Easter and other festive holidays. Sanitary cordons were also established whenever necessary [6]. Portugal started deconfinement in phases that started only on 4 May.

This disease's global reach and the application of classic forms of disease control through quarantine has reduced individuals' mobility. In conjunction with imposed isolation, these measures can have a dramatic effect on health-related quality of life (HRQoL) and contribute to increased anxiety and depression. Previous research has examined physical activity's effects on depression [7] and confirmed that a lower frequency of vigorous physical exercise is significantly associated with higher rates of diagnosed depression. Other studies have found that depression symptoms and physical inactivity are correlated with obesity [8] and that HRQoL and physical function play an important role in depression and anxiety [9]. Physical activity is thus considered crucial for a good HRQoL based on the evidence provided by many studies [10-12].

Previous research has also highlighted economic crises' impacts on HRQoL and mental health. These studies have confirmed social support's importance as protection against recessions' adverse effects on mental health [13, 14]. Other researchers have underlined the importance of job-related problems, especially unemployment, as key determinants of and risk factors for mental health-related difficulties [15].

In addition, to limit the community-level spread of COVID-19, access to non-emergency and elective care has been severely restricted during the pandemic [16]. Visits to primary care physicians and outpatient specialists have declined, and many hospitals have postponed or cancelled elective procedures. These measures' uncertain duration can also contribute to increasing levels of anxiety and decreasing levels of HRQoL.

The COVID-19 quarantine was imposed to fight an ambiguous, continuous threat, which can further contribute to intensifying anxiety and exacerbating individuals' fear of the unknown and thus to reducing HRQoL. People may feel frightened for themselves or their relatives and friends. The high volume of information provided by the media and 
Internet may make individuals panic. Recent research has shown that the fear generated by the COVID-19 pandemic can become chronic and onerous and that anxiety, social media exposure and loved ones' risk levels, amongst other factors, can be predictors of higher levels of this fear [17].

According to some authors, the new limitations on daily life and social activities for an unknown time can also contribute to stress and anxiety and, eventually, loss of confidence in life, resulting in mental health problems [18]. Changes in work-life balance; the risk of losing one's job, which for many became quite real during the state of emergency; and uncertainty about the future exacerbated by an emerging crisis may have serious implications for people's mental health. The imposed isolation has also contributed to more conflicts between family members, which has also increased concerns about the possibility of intensified domestic violence, child abuse or conflicts between separated parents over child custody [6].

The prohibition of religious celebrations or community meetings has further made people more vulnerable and increased their need for social support. In addition, the pandemic has decreased non-COVID-19 patients' access to healthcare. Visits to primary care physicians and outpatient specialists have declined, and many hospitals have postponed or cancelled elective procedures. These changes can be extremely stressful and contribute to higher levels of anxiety and a decrease in HRQoL. Therefore, quarantine measures may have a negative psychological impact on individuals.

With much of the world at home and with the prospect of new waves of the virus and home confinement possibly being decreed again, in-depth analyses are needed of what people felt at that time to understand what effect the quarantine had on their HRQoL. Since this phenomenon has appeared quite suddenly, the authorities have had to take steps quickly to deal with this situation. The global lockdown is an entirely new situation, so, naturally, few previous studies have dealt with this subject. Research has been published on COVID-19's impact on the HRQoL of residents in China [19], as well as another study on possible COVID-19 patients in Vietnam [20] and various other investigations of the pandemic's effects on individuals' mental health and psychological status [18, 21-23]. However, no research has been published, to the best of our knowledge, on COVID-19's impact on HRQoL, mental health and anxiety levels among European populations. Furthermore, none of the aforementioned studies were conducted entirely during the mandatory home quarantine period, so the present research is the first to deal with this kind of data.

This study sought to assess the HRQoL and anxiety levels of individuals in Portugal during the mandatory home quarantine related to the pandemic. A primary objective was to compare the results with the general Portuguese population's
HRQoL before the COVID-19 outbreak (hereafter referred to as pre-COVID-19 pandemic). The research also aimed to understand the factors that can influence the HRQoL of individuals forced into quarantine during the ongoing pandemic.

\section{Methods}

Given the above objectives, a quantitative approach was chosen as the best approach to carrying out this empirical study because this type of methodology is more pragmatic and better able to address the defined research questions. Mandatory social distancing and isolation measures were imposed by the Portuguese authorities to minimise close contact between residents in order to reduce COVID-19's spread, so a face-to-face survey would have been difficult to conduct during the quarantine period. Thus, an online questionnaire was distributed to collect the data, which is also usually associated with faster response times [24].

\section{Questionnaire}

The data collection instrument included 20 questions designed to gather data on quarantined residents' sociodemographic characteristics, feelings, duties, activities, levels of anxiety and HRQoL during the quarantine. The Generalized Anxiety Disorder Seven-item (GAD-7) scale was selected to assess respondents' symptoms, while the 5-level EQ-5D version (EQ-5D-5L) was used to measure HRQoL. These two scales are discussed briefly below. They were included in the two first sections of the questionnaire. The third section comprised a set of questions assessing the participants' feelings, duties and activities during the quarantine. Finally, the respondents' sociodemographic characteristics were covered by nine questions in Portuguese about aspects such as age, gender and education. The edited questionnaire was administered via an online survey platform. The questionnaire's introduction informed the participants that filling in the questionnaire was regarded as their consent to participate in the study.

\section{GAD-7}

The GAD-7 scale is a brief self-report questionnaire previously shown to provide valid assessments of generalised anxiety symptoms in various clinical settings and among the general population [25]. This instrument has been used as both a screening tool and severity measure with patients with GAD [25, 26]. The scale comprises seven items [25]:

(1) Feeling nervous, anxious or on edge

(2) Not being able to stop or control worrying

(3) Worrying too much about different things 
(4) Having trouble relaxing

(5) Being so restless that it is hard to sit still

(6) Becoming easily annoyed or irritable

(7) Feeling afraid as if something terrible might happen

The items correspond to symptoms based on the criteria for GAD. The GAD-7's recall period is the two previous weeks, and the questions are answered on a 4-point Likert scale. The GAD-7 index is calculated by assigning scores of $0,1,2$ and 3 to the response categories of 'not at all', 'several days', 'more than half the days' and 'nearly every day', respectively, and adding together the scores for the 7 questions. Scores of 5, 10 and 15 are taken as the cut-off points for mild, moderate and severe anxiety, respectively. GAD-7 has been found in recent years to be a reliable, valid questionnaire in different languages, including Portuguese [27]. The present study used the Portuguese version of GAD-7 [27].

\section{EQ-5D-5L}

The EQ-5D-5L was introduced by the EuroQol Group in 2009 to improve the EQ-5D 3-level's (3L) sensitivity and reduce its ceiling effect [28]. The EQ-5D-5L encompasses the EQ-5D descriptive system and EQ visual analogue scale. The descriptive system comprises five dimensions: mobility, self-care, usual activities, pain and/or discomfort and anxiety and/or depression. The EQ-5D-5L retains the original 5 dimensions of the EQ-5D-3L, but the number of levels in each dimension was increased from 3 to 5 (i.e. no, slight, moderate, severe and extreme problems), thereby defining a total of 3125 health states [28].

Respondents are asked to indicate their state of health by selecting the most appropriate statement in each dimension and thus providing a five-digit number that describes these individuals' health state [29]. The five-digit code is then converted to a single summary number (i.e. index value) that reflects how good or bad the respondents' state of health is according to the previously documented preferences of the relevant country and/or region's general population. The present study used the Portuguese version of the EQ-5D-5L, and the index was computed using the Portuguese value set [30]. The EQ-5D-5L has been proven to be valid, reliable and responsive in numerous conditions and populations and has been used to examine different patient groups [31, 32] or population subgroups' HRQoL [12,33], including their anxiety levels [34-36].

\section{Sample and data collection}

The current research's target population was the general adult population over the age of 18 residing in Portugal. The lack of a database for this population meant the research team had to conduct a non-probabilistic survey to collect primary data. A combination of convenience and snowball sampling methods was applied to locate residents in Portugal who were either quarantined or self-isolated at home.

A pre-test of the questionnaire was conducted in the last week of March 2020 to collect comments and feedback about the questionnaire from a sample of ten residents living in the Algarve region, who were not included in the main survey. An invitation to take the survey was sent to potential participants by email. The pre-test respondents were asked not only to answer the survey but also to make comments on the survey's content and structure. Based on the results, the questionnaire's introduction was clarified, and ambiguous wording in a couple of questions was rewritten. This study's design was reviewed and approved by the University of Algarve's Research Ethics Committee (i.e. ref. 005/2020).

To approach residents in Portugal more effectively and extensively, the questionnaire was sent individually to acquaintances through the researchers' social networks and published on the Science4COVID-19 website (see https:// www.science4covid19.pt/en/). This website is an initiative of the Portuguese Foundation for Science and Technology and the Agency for Clinical Research and Biomedical Innovation, which was launched 3 April 2020, in partnership with public and private health authorities and scientific research institutions. The website's goal is to mobilise scientific communities to conduct joint research and development projects and activities aimed at combating COVID-19.

The online survey was conducted from 29 March to 19 April 2020, and a total of 904 valid, completed questionnaires were obtained. Since the sample did not match the target population's profile in terms of gender, age and education, the data were weighted according to these variables to increase the results' representativeness for adults residing in Portugal. Data from the Portuguese census [37] were used to compute the weights and compensate for overrepresented and underrepresented subpopulations in the sample as defined by gender, age and education.

To facilitate a comparison with Portugal's pre-COVID-19 pandemic general population, the dataset also included information on all the individuals in a representative random sample of adult Portuguese, which was stratified by gender, age and region. This sample of 1006 individuals was surveyed between November 2015 and January 2016. Further details of the previous study's methodology can be found elsewhere [38].

\section{Data analysis}

Descriptive data analysis (i.e. mean, standard deviation [SD], frequencies and proportions [\%]) was conducted to profile the present sample and examine the respondents' feelings, duties and activities while quarantined. Descriptive analysis 
was also carried out using the GAD-7 and EQ-5D-5L instruments to estimate the study population's level of anxiety and HRQoL. The EQ-5D-5L values were compared to the Portuguese population's norms (i.e. reference values), for which EQ-5D-5L data were collected in the aforementioned cross-sectional study [38].

Correlations between the EQ-5D-5L and GAD-7 scores were evaluated using Spearman's rank correlation coefficient $(\rho)$. In addition, differences between groups defined by sociodemographic variables were evaluated based on non-parametric tests because the EQ-5D-5L index does not approximate the normal distribution. The Mann-Whitney $U$ (i.e. two groups) and Kruskal-Wallis $H$ (i.e. more than two groups) tests were considered to be the most appropriate in this context.

A generalised linear model (GLM) with a Poisson distribution and log link [39] was also used to examine determinants of HRQoL during the COVID-19 quarantine period. This step included investigating the relationships between a set of occupational and attitudinal variables, as well as sociodemographic variables and EQ-5D-5L utility scores. Since the Poisson family of the GLM is only defined for dependent variables that are non-negative integers [40, $p$. 321], the most common approach is to use the EQ-5D-5L disutility score (i.e. a 1 index score) as a dependent variable $[41,42]$, so this procedure was also followed in the present study. The GLM model was also chosen because it accommodates the skewed distribution and heteroscedasticity of the EQ-5D-5L index scores [43, 44].

The literature on this topic reports evidence that sociodemographic variables such as gender, age, education, marital status, employment status and medical condition explain the EQ-5D-5L index score [41, 42]. Thus, two models were estimated. Model 1 included only the above set of sociodemographic variables. Model 2 included additional explanatory variables that we hypothesised also influenced the HRQoL during the COVID-19 quarantine, after controlling for the variables already included in the first model. The variables of household size, place of residence, job-related working and/or studying from home, living with people at risk, supporting other individuals at risk, religion and anxiety level were explored in the second model.

Individuals belonging to smaller households and living in small towns or villages were expected to report higher EQ5D-5L disutility scores (i.e. a poorer state of health). The assumption was also made that individuals living with people at risk, supporting other individuals at risk, and reporting higher levels of anxiety level would have higher disutility scores. Finally, people affiliated with a religion and with occupations allowing them to work and/or study from home were expected to present lower disutility scores.

The level of statistical significance was set at 0.10 , because this study is not grounded in a sample with small size. The use of a threshold of 0.10 does not impact the identification of the key effects in this study, because is it known that any effect, no matter how tiny, can produce a small $p$ value if the sample size or measurement precision is high enough, and large effects may produce unimpressive $p$ values if the sample size is small or measurements are imprecise [45]. Data analyses were performed using IBM SPSS Statistics 26 and Stata 14 software.

\section{Results}

\section{Sample profile}

A total of 915 respondents filled in the online survey. Twelve questionnaires with incomplete data were excluded from the analyses. The final sample thus included 904 valid, completed questionnaires. The respondents' sociodemographic characteristics are displayed in Table 1.

The results show that the majority of respondents were female $(72.9 \%)$, married or living with a partner $(53.6 \%)$ and employed $(67.5 \%)$. The majority also had a high level of education $(62.4 \%)$. In terms of age, respondents between 45 and 59 years old were the largest group in the sample $(31.1 \%)$, followed by those $30-44$ years old $(29.4 \%)($ mean $=41.8 ; \mathrm{SD}=15.4)$. Most respondents were living in households with $2-4$ members $(80.0 \%)$, and only $8.8 \%$ of the participants reported living alone $($ mean $=3.1$; $\mathrm{SD}=1.28)$. The majority lived in a city $(67.2 \%)$, reported having no chronic disease $(75.3 \%)$ and had religious beliefs $(62.7 \%)$. Of the latter, the majority referred to the Roman Catholic religion. About 35\% of respondents selfreported a chronic disease. Finally, roughly one-fifth of the participants said they lived in the same house with family members who belong to the COVID-19 risk group (i.e. over 70 years old), and one-fourth provided support (e.g. shopping for food and medication) to these family members.

As Table 1 above reveals, some population groups were over- and underrepresented. To compensate, data from the Portuguese census [37] were used to weight these subgroups and bring the sample closer to the general Portuguese population in terms of gender, age and education.

Despite a great diversity in the respondents' opinions about what they value most in terms of their way of life, the results presented in Fig. 1 show that over half of the participants affirmed that they 'value a healthy life, regardless of [their] life span' (54.8\%). In contrast, only 5.1\% affirmed that they 'value a long life, regardless of [their] quality of life'. Although a large proportion of respondents had a clear opinion about their way of life, one-sixth were unable to report a preference for one option. 
Table 1 Sample characteristics

\begin{tabular}{|c|c|c|c|}
\hline Characteristic & Category & $n$ & $\%$ \\
\hline \multirow[t]{2}{*}{ Gender } & Male & 245 & 27.1 \\
\hline & Female & 659 & 72.9 \\
\hline \multirow[t]{4}{*}{ Age group } & $18-29$ & 237 & 26.2 \\
\hline & $30-44$ & 266 & 29.4 \\
\hline & $45-59$ & 281 & 31.1 \\
\hline & $60+$ & 120 & 13.3 \\
\hline \multirow[t]{3}{*}{ Education } & Low & 34 & 3.8 \\
\hline & Medium & 306 & 33.8 \\
\hline & High & 564 & 62.4 \\
\hline \multirow[t]{4}{*}{ Marital status } & Single & 331 & 36.6 \\
\hline & Married/living with a partner & 485 & 53.6 \\
\hline & Divorced/separated & 72 & 8.0 \\
\hline & Widowed & 16 & 1.8 \\
\hline \multirow[t]{5}{*}{ Occupational status } & Employed/self-employed & 610 & 67.5 \\
\hline & Unemployed & 21 & 2.3 \\
\hline & Retired/pensioner & 77 & 8.5 \\
\hline & Student & 188 & 20.8 \\
\hline & Homemaker & 8 & 0.9 \\
\hline \multirow[t]{3}{*}{ Place of residence } & City & 608 & 67.2 \\
\hline & Small town & 139 & 15.4 \\
\hline & Small village & 157 & 17.4 \\
\hline \multirow[t]{4}{*}{ Household size } & 1 & 80 & 8.8 \\
\hline & 2 & 226 & 25.0 \\
\hline & $3-4$ & 497 & 55.0 \\
\hline & $5+$ & 101 & 11.2 \\
\hline \multirow[t]{2}{*}{ Religious beliefs } & Yes & 567 & 62.7 \\
\hline & No & 337 & 37.3 \\
\hline \multirow[t]{4}{*}{ Believer's religion } & Roman Catholic & 483 & 86.9 \\
\hline & Christian Orthodox & 21 & 3.8 \\
\hline & Protestant/Evangelical & 16 & 2.9 \\
\hline & Other & 36 & 6.4 \\
\hline \multirow[t]{2}{*}{ Chronic disease } & Yes & 319 & 35.3 \\
\hline & No & 585 & 64.7 \\
\hline \multirow[t]{2}{*}{ Living with a family member at risk } & Yes & 184 & 20.4 \\
\hline & No & 720 & 79.6 \\
\hline \multirow[t]{2}{*}{ Supporting a family member at risk } & Yes & 231 & 25.6 \\
\hline & No & 673 & 74.4 \\
\hline
\end{tabular}

\section{Activities while quarantined}

As shown in Table 2, the two main reasons for being quarantined at home were 'providing care for children' (52.2\%) and 'doing telework' (45.8\%). In addition, $28.4 \%$ of the sample referred to be quarantined because of 'attending video conference classes', but $58.1 \%$ gave other reasons. Table 2 also reveals that more than more than half of the respondents spent their time during the quarantine at home chatting with friends $(64.3 \%)$, consulting social networks $(63.5 \%)$, watching television $(62.4 \%)$, tidying up the house $(59.3 \%)$ and cooking $(58.1 \%)$. Only $11.7 \%$ of the participants reported doing a physical outdoor activity, which was expected since, during the quarantine period, the authorities strongly recommended staying at home. People were only allowed to go outside to exercise as long as this was not done with a group. Notably, less than one-quarter of the sample referred to spending time doing physical activities such as handicraft projects $(20.6 \%)$ or gardening $(23.5 \%)$.

\section{Anxiety and quality of Life}

Table 3 presents the relative frequency distribution by EQ5D-5L dimensions. These results show that the percentage 
Fig. 1 Value of alternative ways of life

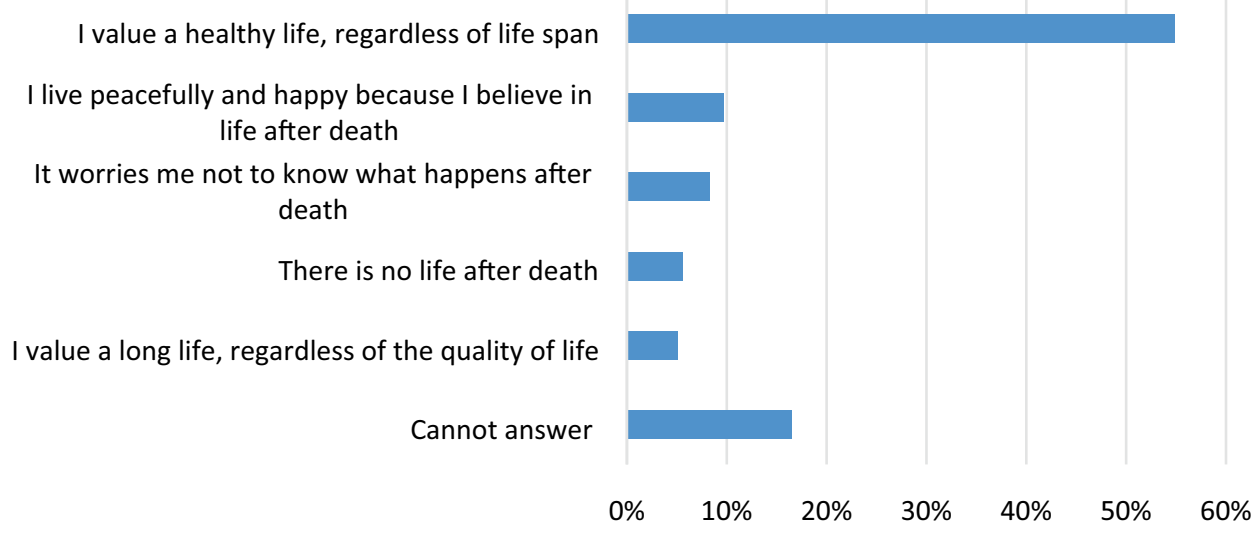

Table 2 Reasons for being quarantined and activities during quarantine $(\%)$

\begin{tabular}{lll}
\hline & Yes & No \\
\hline Reasons for being quarantined & & \\
Doing telework & 45.8 & 54.2 \\
Attending video conference classes & 28.4 & 71.6 \\
Providing care for children & 52.2 & 47.8 \\
Other reasons & 58.1 & 41.9 \\
Ways of spending time during quarantine & & \\
Chatting with friends (e.g. by phone, social & 64.3 & 35.7 \\
$\quad$ media and email) & & \\
Consulting social networks & 63.5 & 36.5 \\
Watching television & 62.4 & 37.6 \\
Tidying up the house & 59.3 & 40.7 \\
Cooking & 58.1 & 41.9 \\
Doing indoor physical activities & 40.9 & 59.1 \\
Working/attending classes & 40.8 & 59.2 \\
Reading & 36.0 & 64.0 \\
Gardening & 23.5 & 76.5 \\
Doing handiwork projects & 20.6 & 79.4 \\
Doing outdoor physical activities & 11.7 & 88.3 \\
\hline
\end{tabular}

Weighted sample according to the Portuguese population data published by INE

of respondents who selected 'no problems' from the EQ5D-5L dimensions were $82.0 \%$ in the dimension of mobility, $85.2 \%$ in self-care, $70.4 \%$ in usual activities, $62.3 \%$ in pain and/or discomfort and $40.7 \%$ in anxiety and/or depression. Although the most frequently reported answer for all the dimensions was 'no problems', less than half of the sample indicated this level in the anxiety and/or depression dimension. In this area, $37.6 \%$ of the participants selected 'slight problems', $14.2 \%$ 'moderate problems' and $6.6 \%$ 'extreme problems' with anxiety and/or depression.

The results for the latter dimension are not only significantly different compared with the other dimensions but different when compared with the general Portuguese population's reference values (i.e. norms). Table 3 above also lists the general population's pre-COVID-19 pandemic norms for the EQ-5D-5L dimensions' relative frequencies, in which a high percentage of the respondents reported 'no problems' in mobility, self-care, usual activities and anxiety and/or depression. The percentage of the study population reporting problems in this dimension was lower than that of the population quarantined for COVID-19. That is, $24.4 \%$ selected 'slight problems', $11.3 \%$ 'moderate problems' and $1.4 \%$ 'extreme problems' in anxiety and/or depression. These results support the conclusion that the residents' isolation during the pandemic crisis generated higher levels of anxiety than those experienced before the crisis.

In terms of HRQoL, the mean EQ-5D-5L index score of the sample during the quarantine is 0.861 (standard error $[\mathrm{SE}]=0.027$ ), which is lower and significantly different than the pre-COVID-19 pandemic general Portuguese population's mean utility score $(0.887 ; \mathrm{SE}=0.005)$. The mean GAD-7 index score is $7.3(\mathrm{SE}=0.58)$, which reveals the respondents' experienced a mild level of anxiety during the quarantine. Table 4 shows that levels of anxiety are significantly and inversely correlated with HRQoL $(\rho=-0.441$; $p<0.001$ ), which means that the respondents with higher levels of anxiety tend to report lower HRQoL and vice-versa.

The GAD-7 index score is significantly and positively correlated with all EQ-5D-5L dimensions, except with mobility. The strongest correlation is between the GAD-7 index score and the EQ-5D-5L's anxiety and/or depression dimension $(\rho=0.611 ; p<0.001)$. These results suggest that adult residents in Portugal suffered from mild anxiety during the quarantine period, which may have decreased their HRQoL. To explore this possible explanatory factor for HRQoL, a regression model was estimated.

Table 5 presents the GAD-7 and EQ-5D-5L mean index scores for the study population by sociodemographic characteristics. The results reveal that statistically significant differences appear in both index scores (i.e. GAD-7 for anxiety and EQ-5D-5L for HRQoL) amongst groups defined by 
Table 3 Distribution of relative frequencies by EQ-5D-5L dimensions $(\%)$

\begin{tabular}{|c|c|c|c|}
\hline Dimension & Level & $\begin{array}{l}\text { Under COVID-19 } \\
\text { quarantine }\end{array}$ & Pre-COVID-19 pandemic \\
\hline \multirow[t]{5}{*}{ Mobility } & No problems & 82.0 & 74.9 \\
\hline & Slight problems & 7.3 & 11.6 \\
\hline & Moderate problems & 3.8 & 9.8 \\
\hline & Severe problems & 4.2 & 3.1 \\
\hline & Extreme problems & 2.7 & 0.6 \\
\hline \multirow[t]{5}{*}{ Self-care } & No problems & 85.2 & 91.2 \\
\hline & Slight problems & 7.5 & 4.3 \\
\hline & Moderate problems & 1.6 & 3.9 \\
\hline & Severe problems & 3.0 & 0.3 \\
\hline & Extreme problems & 2.7 & 0.3 \\
\hline \multirow[t]{5}{*}{ Usual activities } & No problems & 70.4 & 75.9 \\
\hline & Slight problems & 15.3 & 10.5 \\
\hline & Moderate problems & 10.6 & 10.0 \\
\hline & Severe problems & 0.6 & 2.8 \\
\hline & Extreme problems & 3.1 & 0.8 \\
\hline \multirow[t]{5}{*}{ Pain/discomfort } & No problems & 62.3 & 45.9 \\
\hline & Slight problems & 24.5 & 34.2 \\
\hline & Moderate problems & 10.0 & 15.3 \\
\hline & Severe problems & 3.2 & 4.0 \\
\hline & Extreme problems & 0.0 & 0.6 \\
\hline \multirow[t]{5}{*}{ Anxiety/depression } & No problems & 40.7 & 60.9 \\
\hline & Slight problems & 37.6 & 24.4 \\
\hline & Moderate problems & 14.2 & 11.3 \\
\hline & Severe problems & 0.9 & 2.0 \\
\hline & Extreme problems & 6.6 & 1.4 \\
\hline EQ-5D-5L index (SE) & & $0.861(0.027)$ & $0.887(0.005)$ \\
\hline
\end{tabular}

Weighted sample according to the Portuguese population data published by INE

\begin{tabular}{lllllll}
\hline & \multicolumn{2}{l}{ EQ-5D-5L } & & & & \\
\cline { 2 - 6 } & Index score & Mobility & Self-care & Usual activities & Pain/discomfort & Anxiety/depression \\
\hline GAD-7 & $-0.441^{* * *}$ & 0.007 & $0.077^{* * * *}$ & $0.172^{* * *}$ & $0.245^{* * *}$ & $0.611^{* * *}$ \\
\hline$* * * p<0.01$ & & & & & \\
\hline
\end{tabular}

Table 4 Correlation between anxiety and quality of life that levels of anxiety decrease with education (lower $=7.73$ vs higher $=6.43$ ) and HRQoL improves (lower $=0.826$ vs higher $=0.929)$. Strong differences additionally appear in perceived levels of anxiety and HRQoL according to marital status. Moderate levels of anxiety were reported by individuals who were divorced and/or separated (10.26) or widowed (11.68), while individuals with other marital status reported mild anxiety levels (single $=7.70$ vs married $=6.35$ ).

Regarding HRQoL, a better state of health was registered by respondents who were married and/or living with a partner (0.888) or widowed (0.925). Single individuals reported the poorest state of health (0.797), and this was significantly lower than that found for the pre-COVID-19 outbreak period. The GAD-7 index showed the highest level 
Table 5 Anxiety and quality of life by sociodemographic characteristics

\begin{tabular}{|c|c|c|c|c|}
\hline \multirow[t]{2}{*}{ Characteristic } & \multicolumn{2}{|c|}{ GAD-7 index } & \multicolumn{2}{|c|}{ EQ-5D-5L index } \\
\hline & Mean & SE & Mean & SE \\
\hline \multicolumn{5}{|l|}{ 1. Gender***/*** } \\
\hline Male & 5.00 & 0.316 & 0.943 & 0.013 \\
\hline Female & 9.36 & 1.054 & 0.789 & 0.050 \\
\hline \multicolumn{5}{|l|}{ 2. Age group $* * * / * *$} \\
\hline $18-29$ & 7.00 & 0.313 & 0.916 & 0.007 \\
\hline $30-44$ & 7.39 & 1.552 & 0.887 & 0.043 \\
\hline $45-59$ & 6.31 & 0.666 & 0.852 & 0.040 \\
\hline $60+$ & 8.23 & 1.143 & 0.815 & 0.073 \\
\hline \multicolumn{5}{|l|}{ 3. Education $* * * / * * *$} \\
\hline Low & 7.73 & 0.924 & 0.826 & 0.044 \\
\hline Medium & 6.80 & 0.274 & 0.911 & 0.006 \\
\hline High & 6.43 & 0.206 & 0.929 & 0.005 \\
\hline \multicolumn{5}{|l|}{ 4. Marital status $* * / *$} \\
\hline Single & 7.70 & 1.644 & 0.797 & 0.076 \\
\hline Married/living with a partner & 6.35 & 0.429 & 0.888 & 0.026 \\
\hline Divorced/separated & 10.26 & 1.648 & 0.826 & 0.061 \\
\hline Widowed & 11.68 & 2.176 & 0.925 & 0.016 \\
\hline \multicolumn{5}{|l|}{ 5. Occupational status $* * * / * * *$} \\
\hline Employed/self-employed & 7.69 & 0.983 & 0.885 & 0.027 \\
\hline Unemployed & 5.46 & 0.450 & 0.839 & 0.005 \\
\hline Retired/pensioner & 6.40 & 0.798 & 0.814 & 0.093 \\
\hline Student & 7.14 & 0.386 & 0.912 & 0.008 \\
\hline Homemaker & 9.08 & 1.666 & 0.742 & 0.131 \\
\hline \multicolumn{5}{|l|}{ 6. Place of residence*/** } \\
\hline City & 8.06 & 0.914 & 0.857 & 0.041 \\
\hline Small town & 5.70 & 0.297 & 0.900 & 0.019 \\
\hline Small village & 6.45 & 0.984 & 0.852 & 0.052 \\
\hline \multicolumn{5}{|l|}{ 7. Household size*/* } \\
\hline 1 & 7.09 & 0.889 & 0.940 & 0.008 \\
\hline 2 & 8.44 & 0.891 & 0.844 & 0.040 \\
\hline $3-4$ & 6.56 & 0.933 & 0.859 & 0.048 \\
\hline $5+$ & 6.22 & 0.507 & 0.919 & 0.014 \\
\hline \multicolumn{5}{|l|}{ 8. Religious beliefs***/- } \\
\hline Yes & 7.73 & 0.816 & 0.847 & 0.037 \\
\hline No & 5.96 & 0.472 & 0.906 & 0.025 \\
\hline \multicolumn{5}{|l|}{ 9. Chronic disease $-/ * * *$} \\
\hline Yes & 7.44 & 0.772 & 0.763 & 0.062 \\
\hline No & 7.23 & 0.778 & 0.915 & 0.019 \\
\hline \multicolumn{5}{|c|}{ 10. Living with a family member at risk-/- } \\
\hline Yes & 8.72 & 2.356 & 0.729 & 0.093 \\
\hline No & 6.95 & 0.412 & 0.895 & 0.019 \\
\hline \multicolumn{5}{|c|}{ 11. Supporting a family member at risk-/- } \\
\hline Yes & 8.29 & 1.869 & 0.828 & 0.055 \\
\hline No & 6.97 & 0.532 & 0.873 & 0.032 \\
\hline
\end{tabular}

Weighted sample according the Portuguese population data published by INE

$S E$ standard error

$* * * p<0.01, * * p<0.05, * p<0.10$ for the GAD-7/EQ-5D-5L index score. Comparisons of the GAD-7 score and the EQ-5D-5L index score distributions by variables $1,8,9,10$ and 11 were analyzed using the Mann-Whitney $U$ test. All other differences amongst groups were analyzed with the Kruskal-Wallis $H$ test 
of anxiety in the homemaker group (9.08), with the EQ$5 \mathrm{D}-5 \mathrm{~L}$ index revealing a worse perceived HRQoL in the same group (0.742). Notably, employed individuals, who are the largest group, reported medium levels of anxiety (7.69) and HRQoL (0.885). Table 5 above further confirms that GAD-7 and EQ-5D-5L mean index scores also differ significantly by place of residence. For instance, people who live in small towns revealed the lowest levels of anxiety (5.70) and the best HRQoL (0.900) during the quarantine period.

In addition, individuals living in small households (1 member $=7.09$ vs 2 members $=8.44$ ) reported higher levels of anxiety than others did (3-4 members $=6.56$ vs $5+$ members $=6.22$ ). However, this pattern does not hold true for HRQoL since people living both alone and in big households declared a better state of health $(1$ member $=0.940$ vs $5+$ members $=0.919$ ). The GAD-7's mean index scores differ significantly by religious beliefs. Respondents who had religious beliefs felt more anxious (7.73) than those who did not have these beliefs (5.96). Individuals with chronic diseases reported lower levels of HRQoL (0.763) than those who did not have a chronic health condition (0.915). Finally, living with a family member at risk or supporting another individual at risk contributed to a higher level of anxiety and lower HRQoL although the differences are not statistically significant.

Table 6 presents anxiety and quality of life by reasons for being quarantined and activities during quarantine. Overall, the quarantine contributed to mild anxiety levels. Nevertheless, those who were quarantined for other reasons or who were attending video conference classes reported higher levels of anxiety compared to those who were teleworking or taking care of children. Surprisingly, individuals who were doing telework and taking care of children revealed the lowest levels of anxiety and highest levels of HRQoL.

In general, respondents reported mild anxiety levels, with the exception of a normal level of anxiety among individuals who did handiwork projects during the quarantine. On the one hand, those who spent their time tidying up the house, cooking, consulting social networks and watching television reported higher levels of anxiety. On the other hand, people who passed the time by working and/or attending classes, doing physical outdoor activities or reading reported lower levels of anxiety. With regard to HRQoL, those who spent their time working and/or attending classes, doing handiwork projects, doing physical outdoor or indoor activities, gardening or tidying up the house enjoyed higher levels of HRQoL.

The Poisson regression model's estimated coefficients and average marginal effects (AMEs) are presented in Table 7. The overall analysis of the model's significance suggested that all the regressors are statistically significant at 5\% (Wald test $=p<0.001$ ). As expected, Model 1 confirmed that being female and unemployed and having a chronic disease are statistically significant determinants of higher EQ-5D-5L
Table 6 Anxiety and quality of life by reasons for being quarantined and activities during quarantine

\begin{tabular}{lll}
\hline & $\begin{array}{l}\text { Mean GAD-7 } \\
\text { index (SE) }\end{array}$ & $\begin{array}{l}\text { Mean EQ- } \\
\text { 5D-5L index } \\
\text { (SE) }\end{array}$ \\
\hline Reasons for being quarantined & & \\
Doing telework & $6.63(1.127)$ & $0.934(0.015)$ \\
Attending video conference classes & $7.29(0.355)$ & $0.913(0.009)$ \\
Providing care for children & $5.39(0.465)$ & $0.928(0.021)$ \\
Both doing telework and providing care for children & $5.25(0.235)$ & $0.955(0.004)$ \\
Other reasons & $8.17(1.355)$ & $0.782(0.066)$ \\
Ways of spending time during quarantine & & \\
Chatting with friends (e.g. by phone, social media and email) & $7.32(0.556)$ & $0.832(0.036)$ \\
Consulting social networks & $7.89(0.824)$ & $0.861(0.028)$ \\
Watching television & $7.80(0.888)$ & $0.816(0.041)$ \\
Tidying up the house & $8.36(0.915)$ & $0.873(0.026)$ \\
Cooking & $8.54(0.932)$ & $0.854(0.032)$ \\
Doing indoor physical activities & $7.56(0.651)$ & $0.879(0.026)$ \\
Working/attending classes & $6.59(0.750)$ & $0.919(0.013)$ \\
Reading & $7.02(0.061)$ & $0.768(0.061)$ \\
Gardening & $7.37(0.869)$ & $0.808(0.059)$ \\
Doing handiwork projects & $4.93(0.609)$ & $0.947(0.011)$ \\
Doing outdoor physical activities & $6.51(0.752)$ & $0.901(0.023)$ \\
\hline
\end{tabular}

Weighted sample according to the Portuguese population data published by INE

$S E$ standard error 
Table 7 Poisson regression models of EQ-5D-5L disutility scores (weighted sample)

\begin{tabular}{|c|c|c|c|c|c|}
\hline \multirow[t]{2}{*}{ Variable } & \multicolumn{2}{|l|}{ Model 1} & \multicolumn{3}{|l|}{ Model 2} \\
\hline & Coef. & SE & Coef. & SE & AME \\
\hline \multicolumn{6}{|l|}{ Gender (ref. male) } \\
\hline Female & $1.027 * * *$ & 0.229 & $0.761 * * *$ & 0.222 & 0.105 \\
\hline Age & 0.014 & 0.009 & $0.022 * *$ & 0.009 & 0.003 \\
\hline \multicolumn{6}{|l|}{ Education (ref. low) } \\
\hline Medium & $-0.538 * * *$ & 0.211 & -0.125 & 0.142 & -0.017 \\
\hline High & $-0.867 * * *$ & 0.210 & $-0.322 * *$ & 0.149 & -0.045 \\
\hline \multicolumn{6}{|l|}{ Marital status (ref. single) } \\
\hline Married/living with a partner & $-1.183 * * *$ & 0.270 & $-0.979 * * *$ & 0.250 & -0.136 \\
\hline Divorced/separated & $-0.801 * *$ & 0.323 & $-0.618 * *$ & 0.267 & -0.086 \\
\hline Widowed & $-1.635 * * *$ & 0.288 & $-1.728 * * *$ & 0.408 & -0.240 \\
\hline \multicolumn{6}{|c|}{ Occupational status (ref. employed) } \\
\hline Unemployed & $1.344 * * *$ & 0.227 & $1.419 * * *$ & 0.259 & 0.197 \\
\hline Retired/pensioner & -0.569 & 0.388 & 0.024 & 0.300 & 0.003 \\
\hline Student & -0.358 & 0.296 & 0.144 & 0.203 & 0.020 \\
\hline Homemaker & 0.155 & 0.322 & 0.018 & 0.292 & 0.002 \\
\hline \multicolumn{6}{|l|}{ Chronic disease (ref. no) } \\
\hline Yes & $1.014 * * *$ & 0.235 & $1.103 * * *$ & 0.169 & 0.153 \\
\hline Household size & & & 0.053 & 0.054 & 0.007 \\
\hline \multicolumn{6}{|l|}{ Residence (ref. city) } \\
\hline Small town & & & 0.227 & 0.176 & 0.031 \\
\hline Small village & & & $0.660 * * *$ & 0.178 & 0.091 \\
\hline \multicolumn{6}{|c|}{ Working/studying from home (ref. no) } \\
\hline Yes & & & -0.172 & 0.141 & -0.024 \\
\hline \multicolumn{6}{|l|}{ Living with people at risk (ref. no) } \\
\hline Yes & & & $0.513 * * *$ & 0.116 & 0.071 \\
\hline \multicolumn{6}{|l|}{ Supporting people at risk (ref. no) } \\
\hline Yes & & & 0.009 & 0.142 & 0.001 \\
\hline \multicolumn{6}{|l|}{ Religious beliefs (ref. no) } \\
\hline Yes & & & -0.171 & 0.116 & -0.024 \\
\hline Anxiety level & & & $0.080 * * *$ & 0.015 & 0.011 \\
\hline Constant & $-3.792 * * *$ & 0.585 & $-5.239 * * *$ & 0.474 & - \\
\hline
\end{tabular}

$S E$ standard error, $A M E$ average marginal effect

Model 1: Akaike Information Criterion $(\mathrm{AIC})=5,934$; Bayesian Information Criteria $(\mathrm{BIC})=721,494$; Wald test: $\chi_{(14)}^{2}=91.4, p<0.001$. Model 2: $\mathrm{AIC}=576 ; \mathrm{BIC}=502,221$; Wald test: $\chi_{(20)}^{2}=473.0, p<0.001$ $* p<0.1 ; * * p<0.05 ; * * * p<0.01$ disutility scores (i.e. a poorer state of health). In addition, the characteristics of medium and high levels of education, as well as being married, divorced or widowed are statistically significant determinants of lower EQ-5D-5L disutility scores. Only age is not statistically significant at a level of $5 \%$ in Model 1.

After controlling for the variables included in Model 1, the explanatory variables that proved significant in Model 2 are residence, living with people at risk and anxiety (all with $p<0.01$ ) (see Table 7 ). The results show that living in a small village and with people at risk are statistically significant determinants of a poorer HRQoL. The second model also suggests that increases in anxiety levels are significantly associated with negative impacts on the respondents' state of health. The AMEs support the conclusion that, for example, people living with family members at risk have on average 0.513 more EQ-5D-5L disutility (i.e. poorer health) than other individuals, after controlling for all variables. The AME of anxiety is also positive, which implies that an increase of one unit in the GAD-7 anxiety score increases the EQ-5D-5L disutility score by 0.011 (i.e. decreases the index score by 0.011). 


\section{Discussion}

This study sought to assess the HRQoL and anxiety levels of people complying with mandatory home quarantine due to the COVID-19 pandemic. The objectives also included identifying factors that can influence these individuals' HRQoL. As expected, people were quarantined at home because they needed to look after children (i.e. schools were closed on 16 March), do telework or attend video conference classes. The results also show that individuals spent their leisure time doing indoor activities, as required by the compulsory quarantine.

The findings reveal that quarantined people reported higher levels of anxiety and lower HRQoL, as measured by the EQ-5D-5L, compared with the pre-COVID-19 pandemic general population and that those with higher levels of anxiety tended to have a lower HRQoL. Females and elderly individuals reported more anxiety and the worst HRQoL. Differences are evident in anxiety and HRQoL levels that are related to marital status, employment status, place of residence, household size, religious beliefs and chronic diseases, as well as living with a family member at risk and supporting another individual at risk.

The GLM's results show that the HRQoL levels during the COVID-19 quarantine could be explained by various occupational, attitudinal and sociodemographic variables. Being female and unemployed, having a chronic disease, living in a small village and with people at risk and feeling anxious are determinants of higher EQ-5D-5L disutility scores. In contrast, having medium and high levels of education and being married, divorced or widowed are statistically significant factors associated with lower EQ5D-5L disutility scores.

These findings are similar to that of previous research in which the strongest perceived anxiety was concentrated mainly among females and a close relationship was detected with the lowest literacy levels [46]. In the present study, many respondents were working from home, and they had to perform other roles: helping children with schoolwork as schools were closed, doing housework such as cleaning and cooking and helping relatives with daily activities such as shopping. Multi-tasking can be stressful and, many times, handled differently according to gender, which can explain differences found in anxiety levels and HRQoL by gender. For example, women react more anxiously to issues related to the multiple roles they have to play and to interpersonal issues. Men react more anxiously to work and/or financial matters [47].

Another aspect that needs to be emphasised is that many people were working from home or taking care of their children. Since schools were closed and staying at home was mandatory, families had to share the same space for
$24 \mathrm{~h}$ a day, every day, during the 2 months of the home quarantine. Thus, from 1 day to the next, homes were turned into offices, schools, nurseries, gymnasiums and playgrounds. In short, residences were put to a multitude of uses, which implied sharing small spaces and which clearly had serious implications that increased anxiety and decreased HRQoL levels.

These results are also similar to those of Wang et al. [48], who conducted an investigation in China at the COVID-19 pandemic's beginning that revealed that 53.8\% of the participants experienced serious psychological problems. The cited authors confirmed that females were at a higher risk of more anxiety than males were. The current findings are similar to other studies of the public's psychological state and related factors during the COVID19 pandemic in China [18]. The cited research's results show that, although a low percentage of individuals felt anxious, $17 \%$ were depressed. In addition, female gender and increasing age were also associated with more anxiety.

Another recent study found an indisputable relationship between quarantined individuals and increased levels of anxiety. In Italy, which was severely affected by the COVID-19 pandemic, research carried out between 18 and 20 March 2020 with a sample of 3452 individuals revealed that all the respondents felt anxious about the pandemic situation. Within this sample, individuals who had been previously diagnosed with other diseases or who were immunocompromised reported a higher level of anxiety, especially women and the elderly [49].

Given the ongoing pandemic, little has been published thus far about its effects on the general population and/or patients' HRQoL. Another quite recent study sought to identify factors affecting HRQoL among Italian patients with primary antibody deficiencies who switched to remote assistance early on in the COVID-19 pandemic [50]. The results show that $42.3 \%$ of these patients were at risk of developing anxiety or depression and that this risk was related to their fear of COVID-19. Similarly, to the present research, the cited authors found that women had lower HRQoL values and that they experienced more fear because of the pandemic.

In China, Zhang and Ma [19] studied the COVID-19 outbreak's immediate impact on Chinese adults' mental health and quality of life in mainland China. The cited research started 1 week after Wuhan was locked down and travel restrictions were imposed by the Chinese government, so the data collection period was relatively similar to that of the current study. In addition, Zhang and Ma's [19] sampling method (i.e. snowball) and data collection mode (i.e. online survey and phone interviews) were almost the same, although their sample was smaller (number $=263$ ). At the time the data were collected, the pandemic was 
more concentrated in Asia, and only a few cases had been diagnosed in the rest of the world.

The cited study's findings reveal that $52.1 \%$ of the participants reported that they felt horrified by and apprehensive about the pandemic despite experiencing no increase in work-related $(69.2 \%)$ or financial stress $(78.3 \%)$. Zhang and $\mathrm{Ma}$ [19] detected a mild impact of stressful events after assessing the extent of traumatic stress, including traumarelated distressing memories and persistent negative emotions resulting from the pandemic. Although the respondents' feelings of horror and apprehension are in accordance with the present research's results, the cited study in China found only a mild impact on levels of traumatic stress due to COVID-19, which diverges from the current research's findings. This divergence can be explained by how the disease outbreak was generally not considered as severe at the time when the cited study was carried out or by how the Chinese participants ignored the pandemic's actual seriousness [19]. The true impact and lethal nature of the pandemic outbreak were still unknown.

This was not the case in the current study since the participants were fully aware of the virus's lethality as they were well informed by the social media of the pandemic's evolution. The Portuguese authorities had also started daily communications about the pandemic's status inside the country, starting with the first confirmed COVID-19 cases in Portugal. Moreover, the Portuguese paid close attention to what was going on in Italy and Spain because they are similar to Portugal in terms of their population's characteristics. In addition, Spain is the only country with which Portugal shares a border, and Spain can be considered Portugal's sibling in many ways. When Zhang and Ma [19] conducted their research, their city was not on lockdown. In contrast, the present study's objective was to gather data while Portugal's lockdown and home quarantine measures were in place. At the time this research was taking place, the majority of European countries were also on lockdown.

A nationwide survey of psychological distress among Chinese people during the COVID-19 outbreak was conducted by Qiu et al. [21]. They collected data in February 2020 , and their results are similar to those of our study. Over a third (35\%) of the respondents experienced psychological distress, and females reported higher psychological distress than males did. Young adults and the elderly also experienced higher levels of distress than other age groups did. These results are in accordance with the current research, which found that individuals aged 60 or more and between 30 and 44 years old reported higher levels of anxiety than other respondents did. The elderly's more intense anxiety can be explained by being part of the group most at risk during the COVID-19 pandemic, while the other age group's higher levels may be due to how they were most likely working from home, taking care of small children and helping with schoolwork.

Xiao et al.'s recently reported findings [22] confirm that isolation during epidemics contributes to the relevant individuals' increased anxiety and stress, indicating the need to improve these people's social capital and mental health during pandemics. Higher levels of anxiety and lower HRQoL is also related to marital status since divorced, widowed or single individuals report more anxiety, according to the cited researchers [22]. If these individuals self-isolated during the outbreak, they often lived alone, so they would be more likely to feel lonely.

In the present study, significant differences were found between inhabitants living in big cities and small towns. In general, individuals living in the latter areas perceived a better quality of life, but these higher values are not always associated with lower levels of GAD. The findings include that individuals who lived in small villages had a quite positive perception of their HRQoL but had a high level of anxiety compared with those in small towns. These results can be explained by how, given any cases of COVID-19 infection, people living outside large urban centres have trouble (1) accessing healthcare, (2) getting screening tests more quickly, (3) finding help with decision making and (4) gathering accurate, clear information. That is, as a rule, Portuguese living in small villages primarily access news primarily through the television. All these factors can contribute to a greater tendency to experience anxiety. In addition, this part of the population has predisposing factors such as advanced age, isolation and weak social support.

Nguyen et al. [20] investigated the potential benefits of health literacy for HRQoL during the COVID-19 pandemic in Vietnam. The cited authors' findings are similar to ours as they found that age, gender, more education, marital status, comorbidities and physical activity can influence HRQoL. However, Nguyen et al. [20] concluded that taking care of children and working from home during the pandemic outbreak could also be a stressful factor that could decrease HRQoL.

Finally, in the current study, religious individuals reported higher levels of anxiety and lower levels of HRQoL, which is line with previous research [51] that reported these believers experience feelings of anger toward, of abandonment or of being punished by God. Religious individuals also have difficulty reconciling their belief in a loving God with the suffering generated by the pandemic and questions about the ultimate meaning and purpose of life, among other issues.

The present study's results contribute to the existing knowledge on this subject in several unique ways. As far as we know, this research is one of the first to investigate the COVID-19 pandemic's impacts on HRQoL and anxiety in a European population. As mentioned previously, two similar 
studies were conducted in China, but none were conducted during the lockdown period.

Second, this study is one of the first to evaluate HRQoL and anxiety levels when the lockdown and home quarantine measures were still in place. The data were collected at that specific time, and individuals were not asked to recall the home quarantine period to answer the questionnaire. This detail is important since that was a period of uncertainty that may have contributed to high levels of anxiety and depression and to low levels of HRQoL. People did not know at that time how long the home quarantine would last nor what would come after the measures were lifted. In particular, residents in Portugal were watching the news everyday with anxiety and fear for their loved ones because the Italian and Spanish people were suffering greatly and thousands were dying every day.

Third, the data collection was initiated 2 weeks after the beginning of the home quarantine for two main reasons. We felt that people should be somewhat used to being quarantined and that they had already developed some ways to deal with this exceptional situation. In addition, the recall period for the GAD-7 is the two previous weeks.

Fourth, the data were gathered during the peak period of the COVID-19 outbreak in Portugal. Portugal's Minister of Health had already announced that the highest number of cases had occurred at the end of March, so this was public knowledge. Last, the research compared the results for the Portuguese during the lockdown with pre-COVID-19 pandemic statistics, thereby facilitating a fuller understanding of the quarantine and COVID-19 pandemic's impacts on anxiety and HRQoL levels.

This study had certain limitations that need to be taken into account. First, the survey respondents were selected based on non-probabilistic convenience and snowball techniques. Even though these methods are useful ways to select a sample at a low cost or locate members of a specific group who are difficult to reach, respectively, neither convenience nor snowball sampling ensures the participants are representative of the wider population. Thus, the first limitation is related to the results' generalisability to the entire Portuguese population. Second, the data were only collected in Portugal. If other countries had been involved in this study, comparisons across countries would be possible, which would add value to the findings. Third, other variables could have been included in the questionnaire, such as whether the respondent had been tested, whether the respondent was temporarily unemployed, for example. These variables could allow to access other factors that could be relevant for the purpose of the study as well. However, as in the majority of the studies, there was a need to balance the duration of the questionnaire, the data collection method and the purpose of the survey. Additionally, at the time of data collection, the number of tested persons in Portugal was very low; therefore, the probability of having anyone tested in our sample was extremely low. Fourth, comparisons with the pre-COVID data were made for the total sample and only for the EQ-5D-5L. It would have been interesting to compare the quarantined sample with pre-COVID data on GAD-7, but there are no such data for the general Portuguese population. Fifth, HRQoL and anxiety were assessed by self-report measures; therefore, the respondents were not assessed for clinical disorders. Sixth, although effects of lockdowns, such as social isolation or reduced time outside home, may lead to depression, depression was not assessed this study. Further research could potentially focus on the effects of the COVID-19 lockdowns on depression.

HRQoL assessments, such as the one reported here, are important during emergency situations, including the COVID-19 pandemic, because these evaluations help identify factors that could impact the general population's HRQoL. The results can also be used to identify people at risk of anxiety and/or depression during the present pandemic. The findings highlight that governments should not only pay attention to disease control but also take health and social measures specifically designed to prevent mental health problems and feelings of loneliness, thereby preventing a decrease in HRQoL.

This study was conducted during Portugal's lockdown and home quarantine. Over time, the pandemic has appeared to be more or less under control in almost all European countries, and the majority are ending the lockdown and home quarantine restrictions and returning to normal life. As time passes, anxiety levels among the public can be expected to fall, and HRQoL will most likely increase until it matches the norms of most populations. However, a deep economic crisis is currently beginning in Europe and the US, which will certainly spread to the rest of the world and have an impact on the general public's HRQoL. Thus, further studies are needed to assess the effects of the economic crisis caused by the COVID-19 pandemic on people's HRQoL.

In addition, given the catastrophic results that this pandemic has caused worldwide but especially in Europe and the US, countries need to be aware that they have to develop a strategic crisis-prevention plan to deal with future pandemics. Various scientists predict further waves of COVID-19 cases in the fall or winter. The above findings suggest that strategic plans and coordination are needed to provide psychological help and assure the general public that all possible measures are being taken to prevent and control the disease. People need to know that medical support and other effective measures are being taken (e.g. social and economic policies) to prevent decreases in their HRQoL and diminish factors that can cause anxiety and mental problems in the future.

If further waves of the pandemic have a high dissemination capacity similar to the first wave, measures may need 
to be taken to contain the pandemic, and people may have to endure confinement again, so the present study's results could help improve plans and decisions. Policymakers can take advantage of the lessons learned during this exceptional situation. National health authorities should publish guidelines not only for how to deal with disease-related emergencies but also for psychological interventions to ensure the general population's mental health and thus good HRQoL levels.

\section{Conclusion}

The World Health Organization's declaration of a pandemic brought with it a set of measures that changed cognitive and behavioural aspects of individuals' lives, in addition to their social and economic context. This crisis will probably have future consequences including changes in many habits deeply embedded in culture, especially with regard to people's physical proximity and social relationships. Without question, the pandemic will have a profound effect on society and affect, directly or indirectly, many individuals' mental health.

Currently, people around the globe are being asked to stay at home. When this is not an option but instead an obligation, being at home can trigger mixed feelings and cause anxiety. Quarantine can cause anxiety and depression especially in people who are completely alone. Difficulties can also be experienced in managing complicated feelings when people, while not alone, have to learn to manage their children's doubts, restlessness, endless energy and fears, as well as elderly relatives' apprehensions. When the house once longed for as a refuge is a prison, the yearning for freedom becomes strong and natural.

This study thus sought to assess the imposed home quarantine or self-isolation's impact on anxiety and HRQoL. The results suggest that those quarantined at home experienced higher levels of anxiety and a lower HRQoL compared with the pre-COVID-19 pandemic population. The findings further include the factors that explain individuals' HRQoL during the COVID-19 quarantine. One of this research's major strengths is that it was conducted during Portugal's essentially complete lockdown.

The results have implications for the near future. Panic and stress were increased by the sudden outbreak, high infection rate, home isolation and fears of being infected or losing loved ones together with the possibility of losing jobs. All the uncertainty about the future can cause and have implications for mental health. In addition, non-COVID-19 patients had decreased access to healthcare, and the social media exposure and changes in work-life balance were also extremely stressful, contributing to higher anxiety and lower HRQoL levels. Overall, health experts believe that more than $70 \%$ of the world's population will most likely need treatment for physical and/or mental health issues related to COVID-19 infections [52].

The present study's findings offer insights of value to health research. The results show which factors can contribute to increasing anxiety levels and decreasing HRQoL during quarantine. This research also highlighted the need for providing appropriate mental healthcare support during pandemics. Governments and health authorities must provide the general population with objective, updated, accurate, yet simple, clear information on the disease's evolution, measures to be taken to protect themselves and others and actions and steps that are being taken. These findings provide information to health authorities and policymakers that can help them prepare strategic plans to deal with future pandemics and make people feel that everything is once again alright.

Acknowledgements Luis N. Pereira and Lara N. Ferreira are thankful for support from the Research Centre for Tourism, Sustainability and Well-being (CinTurs) [FCT Grant Number UIDP/SOC/04020/2020] and from the Centre for Health Studies and Research - University of Coimbra (CEISUC).

Funding No funding was received for conducting this study.

\section{Compliance with ethical standards}

Conflict of interest The authors declare that they have no conflict of interest.

Ethical approval for human subject research All procedures performed in studies involving human participants were in accordance with the ethical standards of the institutional and/or national research committee and with the 1964 Helsinki Declaration and its later amendments or comparable ethical standards. This study was reviewed and approved by the research ethics committee of the University of the Algarve (ref. 005/2020).

Informed consent Informed consent was obtained from all individual participants included in the study. Participants were informed about their freedom for refusal.

\section{References}

1. European Centre for Disease Prevention and Control. (2020). COVID-19 situation update worldwide, as of 26 May 2020. https ://www.ecdc.europa.eu/en/geographical-distribution-2019-ncovcases. Accessed 26 May 2020.

2. International Monetary Fund. (2020). Europe's COVID-19 Crisis and the Fund's Response. https://blogs.imf.org/2020/03/30/ europes-covid-19-crisis-and-the-funds-response/. Accessed 26 May 2020.

3. Memish, Z. A., Ahmed, Q. A., Schlagenhauf, P., Doumbia, S., \& Khan, A. (2020). No time for dilemma: Mass gatherings must be suspended. Lancet Infectious Diseases. https://doi.org/10.1016/ S0140-6736(20)30754-6. 
4. The World Bank. (2020). DataBank. https://data.worldbank.org/ indicator. Accessed 09 September 2020.

5. Decree n. ${ }^{\circ}$ 2-A/2020. Presidency of the Council of Ministers. Republic Diary No. 57/2020, Series I of 2020-03-20: 11-(5)-11(17). https://dre.pt/application/conteudo/130473161. Accessed 09 September 2020.

6. Oliveira, M., \& Fernandes, C. (2020). Managing the coronavirus pandemic in Portugal: A step-by-step adjustment of health and social services. Psychological Trauma: Theory, Research, Practice, and Policy. https://doi.org/10.1037/tra0000879.

7. Bishwajit, G., O'Leary, D. P., Ghosh, S., Yaya, S., Shangfeng, T., \& Feng, Z. (2017). Physical inactivity and self-reported depression among middle-and older-aged population in South Asia: World health survey. BMC Geriatrics, 17(1), 100.

8. Garimella, R. S., Sears, S. F., \& Gehi, A. K. (2016). Depression and physical inactivity as confounding the effect of obesity on atrial fibrillation. American Journal of Cardiology, 117(11), $1760-1764$.

9. Sousa, R. D. D., Rodrigues, A. M., Gregório, M. J., Branco, J. D. C., Gouveia, M. J., et al. (2017). Anxiety and depression in the Portuguese older adults: Prevalence and associated factors. Frontiers in Medicine, 4, 196.

10. Abdelbasset, W. K., Alsubaie, S. F., Tantawy, S. A., Elyazed, T. E., \& Elshehawy, A. A. (2019). A cross-sectional study on the correlation between physical activity levels and health-related quality of life in community-dwelling middle-aged and older adults. Medicine. https://doi.org/10.1097/MD.0000000000014895.

11. Halaweh, H., Willen, C., Grimby-Ekman, A., \& Svantessona, U. (2015). Physical activity and health-related quality of life among community dwelling elderly. Journal of Clinical Medicine Research. https://doi.org/10.14740/jocmr2307w.

12. Stafrin, J., Sullivan, J., Goldman, D., \& Gill, T. (2017). The association between observed mobility and quality of life in the near elderly. PLoS ONE, 12(8), 1-13.

13. Fernandez, A., Garcia-Alonso, J., Royo-Pastor, C., Garrell-Corbera, I., Rengel-Chica, J., et al. (2015). Effects of the economic crisis and social support on health-related quality of life: first wave of a longitudinal study in Spain. British Journal of General Practice, 65(632), 198-203.

14. Christodoulou, N., \& Christodoulou, G. (2013). Financial crises: Impact on mental health and suggested responses. Psychotherapy Psychosomatics. https://doi.org/10.1159/000351268.

15. Ng, K., Agius, M., \& Zaman, R. (2013). The global economic crisis: Effects on mental health and what can be done. Journal of the Royal Society of Medicine. https://doi.org/10.1177/01410 76813481770.

16. Provenzano, D. A., Sitzman, B. T., Florentino, S. A., \& Buterbaugh, G. (2020). Clinical and economic strategies in outpatient medical care during the COVID-19 pandemic. Regional Anesthesia and Pain Medicine. https://doi.org/10.1136/rapm-2020-10164 0.

17. Mertens, G., Gerritsen, L., Duijndam, S., Salemink, E., \& Engelhard, I. (2020). Fear of the coronavirus (COVID-19): Predictors in an online study conducted in March 2020. Journal of Anxiety Disorders. https://doi.org/10.1016/j.janxdis.2020.102258.

18. Wang, Y., Di, Y., Ye, J., \& Wei, W. (2020). Study on the public psychological states and its related factors during the outbreak of coronavirus disease 2019 (COVID-19) in some regions of China. Psychology, Health \& Medicine. https://doi.org/10.1080/13548 506.2020 .1746817$.

19. Zhang, Y., \& Ma, Z. F. (2020). Impact of the COVID-19 pandemic on mental health and quality of life among local residents in Liaoning Province, China: A cross-sectional study. International Journal of Environmental Research and Public Health. https:// doi.org/10.3390/ijerph17072381.
20. Nguyen, H., Nguyen, M., Do, B., Tran, C., Nguyen, T., Pham, K., et al. (2020). People with suspected COVID-19 symptoms were more likely depressed and had lower health-related quality of life: The potential benefit of health literacy. Journal of Clinical Medicine. https://doi.org/10.3390/jcm9040965.

21. Qiu, J., Shen, B., Zhao, M., Wang, Z., Xie, B., \& Xu, Y. (2020). A nationwide survey of psychological distress among Chinese people in the COVID-19 epidemic: Implications and policy recommendations. General Psychiatry. https://doi.org/10.1136/gpsyc h-2020-100213.

22. Xiao, H., Zhang, Y., Kong, D., Li, S., \& Yang, N. (2020). The effects of social support on sleep quality of medical staff treating patients with coronavirus disease 2019 (COVID-19) in January and February 2020 in China. Medical Science Monitor. https:// doi.org/10.12659/MSM.923549.

23. Yao, H., Chen, J., \& Xu, Y. F. (2020). Patients with mental health disorders in the COVID-19 epidemic. Lancet Psychiatry, 7(4), 228-229.

24. Nardi, P. M. (2006). Doing survey research: A guide to quantitative methods (2nd ed.). Boston: Pearson/Allyn \& Bacon.

25. Spitzer, R. L., Kroenke, K., Williams, J. B., \& Löwe, B. (2006). A brief measure for assessing Generalized Anxiety Disorder: The GAD-7. Archives of Internal Medicine, 166(10), 1092-1097.

26. Swinson, R. P. (2006). The GAD-7 scale was accurate for diagnosing generalised anxiety disorder. Journal of Evidence-Based Medicine, 11(6), 184.

27. Sousa, T., Viveiros, V., Chai, M., Vicente, F., Jesus, G., Carnot, M., et al. (2015). Reliability and validity of the Portuguese version of the Generalized Anxiety Disorder (GAD-7) scale. Health and Quality of Life Outcomes. https://doi.org/10.1186/s1295 5-015-0244-2.

28. Herdman, M., Gudex, C., Lloyd, A., Janssen, M., Kind, P., Parkin, D., et al. (2011). Development and preliminary testing of the new five-level version of EQ5D (EQ-5D-5L). Quality of Life Research, 20(10), 1727-1736.

29. EuroQol Research Foundation. (2019). EQ-5D-5L User Guide. https://euroqol.org/publications/user-guides. Accessed 26 May 2020.

30. Ferreira, P. L., Antunes, P., Ferreira, L. N., Pereira, L. N., \& Ramos-Goñi, J. (2019). A hybrid modelling approach for eliciting health states preferences: The Portuguese EQ-5D-5L value set. Quality of Life Research. https://doi.org/10.1007/s11136-01902226-5.

31. Scalone, L., Ciampichini, R., Fagioli, S., Gardini, I., Gaeta, L., Prete, A., et al. (2013). Comparing the performance of the standard EQ-5D 3L with the new version EQ-5D 5L in patients with chronic hepatic diseases. Quality of Life Research. https://doi. org/10.1007/s11136-012-0318-0.

32. Tran, B., Ohinmaa, A., \& Nguyen, L. (2012). Quality of life profile and psychometric properties of the EQ-5D-5L in HIV/ AIDS patients. Health and Quality of Life Outcomes. https://doi. org/10.1186/1477-7525-10-132.

33. Ferreira, L. N., Ferreira, P. L., Ribeiro, F., \& Pereira, L. N. (2016). Comparing the performance of the EQ-5D-3L and the EQ-5D-5L in young Portuguese adults. Health and Quality of Life Outcomes. https://doi.org/10.1186/s12955-016-0491-x.

34. Crick, K., Al Sayah, F., Ohinmaa, A., \& Johnson, J. A. (2018). Responsiveness of the anxiety/depression dimension of the 3- and 5-level versions of the EQ-5D in assessing mental health. Quality of Life Research. https://doi.org/10.1007/s11136-018-1828-1.

35. Sayah, F., Ohinmaa, A., \& Johnson, J. (2018). Screening for anxiety and depressive symptoms in type 2 diabetes using patientreported outcome measures: Comparative performance of the EQ5D-5L and SF-12v2. Medical Decision Making Policy \& Practice. https://doi.org/10.1177/2381468318799361. 
36. Khue, P., Thom, V., Minh, D., Quang, L. M., \& Hoa, L. (2019). Depression and anxiety as key factors associated with quality of life among lung cancer patients in Hai Phong, Vietnam. Frontiers in Psychiatry. https://doi.org/10.3389/fpsyt.2019.00352.

37. Portuguese Statistical Office. (2012). Census 2011 Resultados Definitivos-Portugal. Lisbon: INE.

38. Ferreira, L. N., Ferreira, P. L., Ferreira, P. A., \& Pereira, L.N. (2020). EQ-5D-5L Portuguese population norms. (submitted).

39. Manning, W. G., \& Mullahy, J. (2001). Estimating log models: To transform or not to transform? Journal of Health Economics, 20(4), 461-494.

40. Cameron, A. C., \& Trivedi, P. K. (2010). Microeconometrics using Stata. College Station: Stata Press Publications.

41. McCaffrey, N., Kaambwa, B., Currow, D. C., \& Ratcliffe, J. (2016). Health-related quality of life measured using the EQ5D-5L: South Australian population norms. Health and Quality of Life Outcomes, 14(1), 133.

42. Grochtdreis, T., Dams, J., König, H. H., \& Konnopka, A. (2019). Health-related quality of life measured with the EQ-5D-5L: Estimation of normative index values based on a representative German population sample and value set. European Journal of Health Economics, 20(6), 933-944.

43. de Jong, P., \& Heller, G. Z. (2008). Generalized linear models for insurance data. Cambridge: Cambridge University Press.

44. Manning, W. G., Basu, A., \& Mullahy, J. (2005). Generalized modeling approaches to risk adjustment of skewed outcomes data. Journal of Health Economics, 24(3), 465-488.

45. Wasserstein, R. L., \& Lazar, N. A. (2016). The ASA's statement on p-values: Context, process, and purpose. The American Statistician. https://doi.org/10.1080/00031305.2016.1154108.

46. Luni, F., \& Jawad, A. (2009). Prevalence of depression and anxiety in a village in Sindh. Journal of Ayub Medical College, 21(2), $68-72$.
47. David, A., \& Kessler, R. (1998). Everyday stressors and gender differences in daily distress. Journal of Personality and Social Psychology, 75(3), 670-680.

48. Wang, C., Pan, R., Wan, X., Tan, Y., Xu, L., Ho, C., et al. (2020). Immediate psychological responses and associated factors during the initial stage of the 2019 coronavirus disease (COVID-19) epidemic among the general population in China. International Journal of Environmental Research and Public Health. https:// doi.org/10.3390/ijerph17051729.

49. Barari, S., Caria, S., Davola, A., Falco, P., Fetzer, T., Fiorin, S., et al. (2020). Replication data for: Evaluating COVID-19 public health messaging in Italy: Self-reported compliance and growing mental health concerns. Harvard Dataverse. https://doi. org/10.7910/DVN/1SBQCX.

50. Lee, S. (2020). Coravirus Anxiety Scale: A brief mental health screener for COVID-19 related anxiety. Death Studies. https://doi. org/10.1080/07481187.2020.1748481.

51. Dein, S., Loewenthal, K., Lewis, C., \& Pargament, K. (2020). COVID-19, mental health and religion: An agenda for future research. Mental Health, Religion \& Culture. https://doi. org/10.1080/13674676.2020.1768725.

52. Pulvirenti, F., Cinetto, F., Milito, C., Bonanni, L., Pesce, A., Leodori, G., et al. (2020). Health-related quality of life in common variable immunodeficiency Italian patients switched to remote assistance during the COVID-19 pandemic. Journal of Allergy and Clinical Immunology: In Practice. https://doi.org/10.1016/j. jaip.2020.04.003.

Publisher's Note Springer Nature remains neutral with regard to jurisdictional claims in published maps and institutional affiliations. 